\title{
REPRESENTATIONS OF FUNDAMENTAL GROUPS OF MANIFOLDS WITH A SEMISIMPLE TRANSFORMATION GROUP
}

\author{
ROBERT J. ZIMMER
}

\section{INTRODUCTION}

In this paper, we examine the relationship between the topology of a manifold $M$, specifically the finite dimensional representation theory of the fundamental group $\pi_{1}(M)$, and the Lie groups that can act on $M$. More precisely, let $G$ be a connected semisimple Lie group of higher real rank, and suppose $G$ acts continuously on a (topological) manifold $M$, preserving a finite measure. The main theme of this paper is that the representation theory of $\pi_{1}(M)$ in low dimensions is to a large extent controlled by that of $G$ (the latter of course being well understood). In particular, under natural hypotheses (e.g., that the action of $G$ on $M$ is engaging, i.e., there is no loss of ergodicity in passing to finite covers; see Definition 3.1 below), we prove that if $G$ has no nontrivial representations below dimension $d$, then every representation of $\pi_{1}(M)$ below dimension $d$ is finite; that is, it factors through a finite quotient group. Under different but related hypotheses (namely that the action is topologically engaging, that is, roughly speaking, that the action is proper on the universal cover; see Definition 3.2), we show that $\pi_{1}(M)$ admits no faithful representation over $\overline{\mathbf{Q}}$ below dimension $d$. These results of course impose severe restrictions on the manifolds on which $G$ can act. The hypotheses of engaging or topological engaging are quite mild, and one or the other is satisfied in every known nontrivial example. We also remark that Gromov has shown [5] that every real analytic connection preserving action of $G$ is topologically engaging.

Rather than considering representations, one can consider, more generally, homomorphisms into a general algebraic group, and the above results become special cases of the following theorems. (These results, as well as most of the others in this paper, hold for spaces much more general than the class of topological manifolds. See the beginning of $\S 2$.)

Theorem 5.1. Let $G$ be a connected semisimple Lie group, each of whose simple factors has real rank at least 2 . Suppose $G$ acts continuously on a manifold

Received by the editors May 10, 1988.

1980 Mathematics Subject Classification (1985 Revision). Primary 57S20.

The author's research was partially supported by an NSF Grant. 
$M$, preserving a finite measure, and that the action is engaging. Assume $\pi_{1}(M)$ is finitely generated. Let $H$ be an algebraic group (over $\mathbf{C}$ ), and suppose every Lie algebra homomorphism $\mathfrak{g}_{\mathbf{C}} \rightarrow \mathfrak{h}$ is trivial. Then every homomorphism $h$ : $\pi_{1}(M) \rightarrow H$ is finite.

Theorem 5.2. Let $G$ be a connected semisimple Lie group, each of whose simple factors has real rank at least 2 . Suppose $G$ acts continuously on a manifold $M$, preserving a finite measure, and that the action is topologically engaging. Assume $\pi_{1}(M)$ is finitely generated and infinite. Let $H$ be an algebraic group (over $\overline{\mathbf{Q}}$ ), and suppose every Lie algebra homomorphism $\mathfrak{g}_{\mathbf{C}} \rightarrow \mathfrak{h}$ is trivial. Then there is no faithful homomorphism $h: \pi_{1}(M) \rightarrow H_{\overline{\mathbf{Q}}}$.

Via similar techniques, we can prove a variety of related results under varying hypotheses. For example, if $H$ is defined over $\mathbf{R}$, it is natural to suppose only that there is no nontrivial Lie algebra homomorphism $\mathfrak{g} \rightarrow \mathfrak{h}_{\mathbf{R}}$. It is also natural to consider the case of homomorphisms of $\pi_{1}(M)$ into $p$-adic groups. We obtain satisfactory results in these cases as well, and these are spelled out in $\S 5$. We also consider the case of actions of a lattice subgroup $\Gamma \subset G$. The situation here is more complex, and even the definitions of engaging and topologically engaging actions require more care (cf. §8). For many such $\Gamma$, we have the following cohomological condition $(\mathrm{C}): H^{2}(\Lambda, \mathbf{R})=0$ for every finite index subgroup $\Lambda \subset \Gamma$. Then we show

Theorem 8.5. Let $G$ be as above, and let $\Gamma \subset G$ be a lattice subgroup, satisfying cohomological condition $(\mathrm{C})$. Let $d$ be the minimal dimension of a nontrivial real representation of the Lie algebra $\mathfrak{g}$. Then there is no engaging or topologically engaging action of $\Gamma$ on any compact manifold with an infinite abelian fundamental group of rank strictly less than $d$.

The action of $G$ on $G / \Gamma$ is both engaging and topologically engaging. In this case, Theorems 5.1 and 5.2 follow from Margulis' superrigidity theorem. We can thus view Theorems 5.1 and 5.2 as showing that certain features of lattices in higher real rank semisimple groups are still present in the fundamental group of any manifold on which $G$ acts in an engaging, volume preserving way.

In [14], we examined the holonomy group of a Lie foliation with symmetric spaces as leaves. We can describe this roughly in our present context by saying that under suitable geometric hypotheses on a $G$-action (i.e., the existence of a transverse Lie structure) that the fundamental group of the ambient manifold must have an arithmetic quotient group of a very precise type. Thus, in [14] we assert the existence of a certain type of representation of $\pi_{1}(M)$, while, of course, in the present paper, we show nonexistence of certain types of representations. Gromov has also considered these issues in [5], obtaining, under geometric hypotheses, "lower bounds" on $\pi_{1}(M)$. Gromov's paper makes very clear the importance of the role of properness on the universal cover of $M$, and motivated our definition of topological engaging. 
The author would like to thank A. Ash, A. Borel, K. Corlette, G. Prasad, and T. N. Venkataramana for helpful conversations related to this paper, and M. Gromov for communicating his ideas.

\section{Preliminaries of ERgodic theory}

Our results will hold for actions on any space that has a good theory of covering spaces and good measure theory. Hence, throughout we shall assume $M$ is a connected, locally path-connected, semilocally 1-connected, locally compact, separable, metrizable space, with a finitely generated fundamental group. For brevity, we shall simply call such a space "standard."

We can view the universal covering space of $M$ as a principal $\pi_{1}(M)$-bundle. Any action of a connected group $G$ on $M$ lifts to $\widetilde{M}$ in such a way as to commute with $\pi_{1}(M)$. In other words, $\widetilde{G}$ acts by principal bundle automorphisms of $\widetilde{M}$. For any homomorphism of $\pi_{1}(M)$ into a group $H$, we obtain an associated principal $H$-bundle on which $\widetilde{G}$ will also act by principal bundle automorphisms. If $G$ is semisimple and $H$ is algebraic, there is a great deal known about the measure theoretic structure of such actions [12,13]. Our approach to proving Theorems 5.1 and 5.2 and related results will be to exploit this information. An interesting feature of the argument is the necessity to consider such bundles where the structure group is $p$-adic, even if one is only interested in real or complex representations. The reader familiar with the general theory of representations of finitely generated groups will not find this surprising. Since we will be dealing with the measure theoretic aspects of such bundles, it is convenient to view them as products, which can always be done measurably. The action of $\widetilde{G}$ is then described via a cocycle. We now review some of the basic notions concerning measurable cocycles for group actions. See [13] for a leisurely account with an eye toward geometric applications.

Suppose $G$ and $L$ are locally compact groups and that $M$ is a measure space on which $G$ acts measurably on the right, leaving the measure quasiinvariant. A Borel function $\alpha: M \times G \rightarrow L$ is called a cocycle if for all $g$, $h \in G, \alpha(m, g h)=\alpha(m, g) \alpha(m g, h)$ for (almost) all $m \in M$. Two such cocycles $\alpha, \beta$ are called equivalent or cohomologous (and we write $\alpha \sim \beta$ ) if there is a measurable $\varphi: M \rightarrow L$ such that for each $g \in G$ we have $\varphi(m) \alpha(m, g) \varphi(m g)^{-1}=\beta(m, g)$ a.e. If $P \rightarrow M$ is a principal $L$-bundle on which $G$ acts by principal bundle automorphisms, then under any measurable trivialization $P \cong M \times L$, the action of $G$ on $P$ will be given by $(m, \lambda) g=\left(m g, \alpha(m, g)^{-1} \lambda\right)$ for some cocycle $\alpha$. Choosing a different measurable trivialization is equivalent to choosing an equivalent cocycle. If $H \subset L$ is a closed subgroup, it is of interest to know when $\alpha$ is cohomologous to a cocycle taking all values in $H$. This is equivalent to the assertion that there is a measurable $G$-invariant reduction of $P$ to the group $H$. If $Y$ is a right $L$-space, a measurable $\varphi: M \rightarrow Y$ is called $\alpha$-invariant if $\varphi(m) \alpha(m, g)=\varphi(m g)$. We then have the following simple proposition. 
Proposition 2.1. The following are equivalent.

(i) $\alpha$ is equivalent to a cocycle taking all values in $H$.

(ii) There is an $\alpha$-invariant $\varphi: M \rightarrow H \backslash L$.

The basic information we shall use regarding cocycles for semisimple group actions is given in the following theorem. It is a part of a general superrigidity theorem for cocycles, proved in $[10,11,12]$.

Theorem 2.2. Suppose $G$ is a connected semisimple Lie group of higher real rank, i.e., the R-rank of every simple factor of $G$ is at least 2 . Suppose the action of $G$ on $M$ preserves a finite measure, and let $\alpha: M \times G \rightarrow H$ be a cocycle. Then $\alpha$ is equivalent to a cocycle into a compact subgroup of $H$ under any of the following assumptions.

(i) $H$ is a real or complex algebraic group and there is no Lie algebra homomorphism $\mathfrak{g} \rightarrow \mathfrak{h}$.

(ii) $H$ is the set of $p$-adic points of a p-adic algebraic group.

(iii) $H$ is amenable, and the $G$-action on $M$ is ergodic.

A useful device in the study of cocycles is the notion of the Mackey range of a cocycle [9, 12], which we now recall. Given a cocycle $\alpha: M \times G \rightarrow H$, we let $P=M \times H$ and consider the action of $G$ on $P$ defined by the cocycle, as given above. We let $X$ be the space of ergodic components of the $G$-action. The action of $H$ on $P$ by right translation on the second factor commutes with $G$, and hence we have an action of $H$ on $X$, which will preserve the natural measure class. This $H$-action on $X$ is called the Mackey range of $\alpha$, and it will be ergodic if the $G$-action on $M$ is ergodic. A useful feature of this construction is that $\alpha$ is equivalent to a cocycle taking values in a closed subgroup $H_{1} \subset H$ if and only if there is a measurable $H$-map $X \rightarrow H / H_{1}$. (See [9] for a proof.) Here is a related fact we shall need.

Lemma 2.3. Suppose $\alpha: M \times G \rightarrow H$ is a cocycle and that $\pi: H \rightarrow L$ is a homomorphism. Suppose $Y$ is a right L-space and that there is a $\pi \circ \alpha$ invariant function $\varphi: M \rightarrow Y$. Let $X$ be the Mackey range of $\alpha$. Then there is a measurable $H$-map $X \rightarrow Y$.

Proof. We have the equation $\varphi(m) \pi(\alpha(m, g))=\varphi(m g)$ for all $g, m$. Define $\theta: M \times H \rightarrow Y$ by $\theta(m, h)=\varphi(m) \pi(h)$. This is clearly an $H$-map, and so it suffices to see it factors to $X$; i.e., it suffices to see $\theta$ is $G$-invariant. However,

$$
\begin{aligned}
\theta((m, h) g) & =\theta\left(m g, \alpha(m, g)^{-1} h\right) \\
& =\varphi(m g) \pi\left(\alpha(m, g)^{-1}\right) \pi(h)=\varphi(m) \pi(h)=\theta(m, h) .
\end{aligned}
$$

Suppose now that $N$ is a locally compact separable space on which $G$ acts continuously. We recall that the action is called proper if for any pair of compact subsets $A, B \subset N$, we have $\{g \in G \mid A g \cap B$ nonempty $\}$ has compact closure. An action on a standard Borel space is called tame (or "smooth in the sense of 
ergodic theory") if the quotient space has a countably generated Borel structure $[12,13]$. For $N$ locally compact and separable, this is equivalent to all orbits being locally closed, or equivalently [12] the quotient space is $T_{0}$. Any proper action is tame. If $\mu$ is any quasi-invariant measure on $N$, we call the action $\mu$-tame if there is a $G$-invariant Borel conull set on which the action is tame, $\mu$-proper if it is $\mu$-tame and almost every stabilizer is compact. Thus, a proper action satisfies all these conditions for any $\mu$. For a $\mu$-tame action, the ergodic components are precisely the orbits [12]. For ease of reference, we record a special case.

Lemma 2.4. If $\alpha: M \times G \rightarrow H$ is a cocycle, and the $G$-action on $P=M \times H$ is tame, then the Mackey range of $\alpha$ is the action of $H$ on $P / G$.

Proposition 2.5. (a) Suppose $(N, \nu) \rightarrow M$ is a measurable H-map where the action of $H$ on $M$ is proper. Then the action on $N$ is $\nu$-proper.

(b) Suppose $G \times H$ acts on $N$, and that $G$ and $H$ each act tamely. Let $\mu$ be a $G \times H$ quasi-invariant measure on $N$, and $\mu_{1}, \mu_{2}$ the projections onto $N / G, N / H$, respectively. Then the G-action on $N / H$ is $\mu_{2}$-tame if and only if the $H$-action on $N / G$ is $\mu_{1}$-tame.

Proof. (a) follows easily from [12, Chapter 2] and (b) from the definitions.

\section{ENGAGEMENT}

Suppose $G$ is a connected Lie group acting on a standard space (e.g., a topological manifold with finitely generated fundamental group) $M$. The action lifts to a unique action of $\widetilde{G}$ on $\widetilde{M}$ commuting with $\pi_{1}(M)$. We also have an action of $\widetilde{G}$ (in fact, of some finite covering of $G$ ) on any finite cover $M^{\prime}$ of $M$.

Definition 3.1. Suppose $\mu$ is a quasi-invariant measure for the $G$-action on $M$. The action of $G$ on $M$ is called $\mu$-engaging (or simply engaging if $\mu$ is understood) if there is no loss of ergodicity in passing to finite covers of $M$; more precisely, if for every finite cover $M^{\prime}$, every measurable $\widetilde{G}$-invariant function on $M^{\prime}$ is lifted from a function on $M$.

In particular, if $G$ acts ergodically on $M$, the condition that the action be engaging is simply that $\widetilde{G}$ is ergodic on every finite cover of $M$.

Definition 3.2. The action of $G$ on $M$ is called topologically engaging if there is some $\tilde{g} \in \widetilde{G}$ that acts tamely on $\widetilde{M}$ (i.e., has locally closed orbits, e.g., acts properly) and that projects to an element $g \in G$ that does not lie in a compact subgroup.

Example 3.3. Suppose that $G$ is a semisimple Lie group without compact factors and that we have an embedding $G \rightarrow H$ where $H$ is another semisimple Lie group without compact factors. Suppose $\Gamma \subset H$ is a cocompact discrete irreducible subgroup. Then $G$ acts on $H / \Gamma$ and the action is both engaging 
and topologically engaging. Variants of this construction provide most of the known ergodic volume preserving actions of $G$ on a compact manifold.

Example 3.4. Gromov [5] proves that if $G$ is a semisimple Lie group with no compact factors and finite fundamental group and that if $G$ acts real analytically on $M$ preserving a pseudo-Riemannian metric, then $\widetilde{G}$ acts properly on $\widetilde{M}$. In particular, the action is topologically engaging.

Now let $\Gamma=\pi_{1}(M)$. Since $G$ acts by automorphisms of the principal $\Gamma$ bundle $\widetilde{M} \rightarrow M$, we obtain a cocycle $\alpha: M \times \widetilde{G} \rightarrow \Gamma$. We now consider the relationship of the conditions in Definitions 3.1 and 3.2 with properties of the Mackey range of $\alpha$.

Proposition 3.5. Suppose the action of $G$ on $M$ is engaging. Then $\alpha$ is not equivalent to a cocycle taking values in a subgroup $\Lambda \subset \Gamma$ with the following properties:

(a) $\Gamma / \Lambda$ is infinite;

(b) there is a subgroup $\Lambda_{0} \subset \Lambda$ of finite index that is normal in $\Gamma$ and with $\Gamma / \Lambda_{0}$ residually finite.

Equivalently (by (2.1) the Mackey range $X$ does not admit a measurable $\Gamma$-map $X \rightarrow \Gamma / \Lambda$, where $\Lambda$ satisfies (a) and (b).

Proof. Since $\Gamma / \Lambda_{0}$ is infinite and residually finite, we can choose a finite quotient $\Gamma / \Lambda_{1}, \Lambda_{1} \supset \Lambda_{0}$, whose cardinality is greater than that of $\Lambda / \Lambda_{0}$. Let $p: \Gamma \rightarrow \Gamma / \Lambda_{1}$ be the quotient map. Then the Galois covering of $M$ corresponding to $\Lambda_{1}$ is measurably isomorphic to $M \times \Gamma / \Lambda_{1}$ with the $\widetilde{G}$-action given by the cocycle $p \circ \alpha$. If $\alpha$ takes values in $\Lambda$, then $M \times\left(\Lambda_{1} / \Lambda_{1}\right) \subset M \times \Gamma / \Lambda_{1}$ will be a $\widetilde{G}$-invariant measurable set. Since the cardinality of $\Lambda_{1} / \Lambda_{1}$ is less than that of $\Gamma / \Lambda_{1}$, the characteristic function of this set does not lift from $M$.

Proposition 3.6. Suppose the action of $G$ on $M$ is topologically engaging (and faithful) where $G$ is semisimple without compact factors. Suppose there is a finite $G$-invariant ergodic measure $\mu$ on $M$. Then the $\Gamma$ action on the Mackey range of $\alpha$ is not of the form $\Gamma / F$ where $F$ is a finite subgroup.

Proof. Let $H \subset \widetilde{G}$ be the group generated by $\tilde{g}$. Then $H$ is closed and projects to a subgroup of noncompact closure in $G$. By Moore's theorem [12], $H$ cannot have almost all orbits be periodic, and hence the action of $H$ on $M$ is not $\mu$ tame. (In fact, if $G$ is simple, $H$ will act properly ergodically.) Let $\widetilde{X}$ be the Mackey range of $\tilde{\alpha}=\alpha \mid M \times H$. The action of $H$ on $\widetilde{M}$ is tame by the definition of topological engaging, so $\widetilde{X}=\widetilde{M} / H$. The action of $\Gamma$ on $\widetilde{M}$ is clearly tame with quotient $M$, and hence by Proposition 2.5 (b) the action of $\Gamma$ on $\tilde{X}$ cannot be $\nu$-tame, where $\nu$ is the natural measure on $\tilde{X}$. However, by the definition of the Mackey range, we have a measurable $\Gamma$-map $\widetilde{X} \rightarrow X$. If $X=\Gamma / F$, this would then contradict Proposition 2.5(a).

The first assertion of the following proposition is obvious, and the second follows by routine measure theoretic arguments. 
Proposition 3.7. (a) Suppose $M^{\prime} \rightarrow M$ is a finite cover. If the action of $\widetilde{G}$ on $M$ is engaging, so is the action on $M^{\prime}$. The action on $M$ is topologically engaging if and only if the action on $M^{\prime}$ is as well.

(b) Suppose the action on $M$ is $\mu$-engaging. Let $\mu=\int{ }^{\oplus} \mu_{t}$ be the decomposition of $\mu$ into ergodic measures on $M$. Then for almost all $t$, the $G$-action is $\mu_{t}$ engaging.

\section{REDUCTION TO HOMOMORPHISMS OVER $\overline{\mathbf{Q}}$}

We give a criterion for every homomorphism from a finitely generated group into an algebraic group to be finite. Although one can prove sharper statements, we content ourselves here with one that is easily established and which will suffice for our present purposes.

Theorem 4.1. Let $G$ be a connected algebraic group (over $\mathbf{C}$ ) and $\Gamma$ a finitely generated group. Suppose that for every algebraic group $L$, which is a subquotient of $G$, every $\overline{\mathbf{Q}}$-structure on $L$ and every finite index subgroup $\Lambda \subset \Gamma$, we have that every homomorphism $\Lambda \rightarrow L_{\overline{\mathbf{Q}}}$ is finite. Then every homomorphism $\Gamma \rightarrow G$ is finite.

Proof. Fix $\pi: \Gamma \rightarrow G$, and let $H$ be the connected component of the algebraic hull of the image. By passing to a subgroup of finite index in $\Gamma$, we may assume $H$ is connected and that the image of $\pi$ is Zariski dense in $H$. Let $R$ be the radical of $H$, and $L=H / R$. We first claim that it suffices to see that the image of $\Gamma$ in $L$ is finite. For if this is so, then on a subgroup $\Lambda$ of finite index, we have $\pi(\Lambda) \subset R$. Since $R$ is solvable and $\pi(\Lambda)$ is finitely generated, if $\pi(\Lambda)$ is not finite it will have a subgroup of a finite index that maps onto $Z$. This would obviously contradict the hypotheses. Thus, we shall assume $\pi: \Gamma \rightarrow L$ and that the image is Zariski dense. Since $L$ is semisimple, we may fix a realization of $L \subset \mathrm{GL}(n, \mathbf{C})$ as an algebraic group defined over $\mathbf{Q}$. Since the space of homomorphisms $\Gamma \rightarrow L$ is then a variety over $\mathbf{Q}$, we can approximate any $\pi: \Gamma \rightarrow L$ by a sequence $\pi_{j}: \Gamma \rightarrow L_{\overline{\mathbf{Q}}}$. Since $\pi_{j}(\Gamma)$ is finite, we have $\left|\operatorname{tr}\left(\pi_{j}(\lambda)\right)\right| \leq n$ for all $\lambda \in \Gamma$, and hence $|\operatorname{tr}(\pi(\lambda))| \leq n$ as well. It follows that $\operatorname{tr}(\pi(\lambda)) \in \overline{\mathbf{Q}}$ for all $\lambda$. If not, we can choose $\sigma \in \operatorname{Gal}(\mathbf{C} / \overline{\mathbf{Q}})$ such that $|\sigma(\operatorname{tr}(\pi(\lambda)))|>n$, i.e., $|\operatorname{tr}((\sigma \circ \pi)(\lambda))|>n$, where, as usual, we let $\sigma$ act on complex matrices. Since $L$ is defined over $\mathbf{Q}, \sigma \circ \alpha: \Gamma \rightarrow L$ as well, showing that this is impossible. It follows by a result of Vinberg [12, Lemma 6.1.7] that there is a realization of $L$ as a linear group defined over $\overline{\mathbf{Q}}$ in such a way that $\pi(\lambda) \in L_{\overline{\mathbf{Q}}}$ for all $\lambda \in \Gamma$. By hypothesis, this shows that $\pi$ is finite.

\section{Proof of the Main theorems}

Theorem 5.1. Suppose $G$ is a connected semisimple Lie group each of whose simple factors has real rank at least 2 . Let $H$ be a complex algebraic group such that every homomorphism of Lie algebras $\mathfrak{g}_{\mathbf{C}} \rightarrow \mathfrak{h}$ is trivial. Assume there 
is an action of $G$ on $M$ that preserves a finite measure and is engaging. Then every homomorphism $\pi_{1}(M) \rightarrow H$ is finite.

We remark that the conclusion implies that there are only finitely many conjugacy classes of homomorphisms into a given $H$, and hence that all such homomorphisms factor through a fixed (for a given $H$ ) finite quotient of $\Gamma$.

Proof. By Proposition 3.7(b), we can assume the measure on $M$ is ergodic under $G$. Let $\Gamma=\pi_{1}(M)$. By Theorem 4.1, it suffices to assume that $H$ is defined over $\overline{\mathbf{Q}}$ and to see that any $\pi: \Gamma \rightarrow H_{\overline{\mathbf{Q}}}$ is finite for then this would apply to any finite index subgroup of $\Gamma$ and any subquotient of $H$. We may clearly further assume that $\pi(\Gamma)$ is Zariski dense in $H$. Since $\pi(\Gamma) \subset H_{\overline{\mathbf{Q}}}$ and $\Gamma$ is finitely generated, there is a number field $k([k: \mathbf{Q}]<\infty)$ such that $\pi(\Gamma) \subset H_{k}$. In particular, $H$ is defined over $k$. Let $L=H_{k / \mathbf{Q}}$, the algebraic Q-group obtained from $H$ by restriction of scalars [12]. Every simple factor of $L$ is isomorphic to a simple factor of $H$ (cf. [6]), and hence every local homomorphism $G \rightarrow L$ is trivial as well. We have a natural identification of $H_{k}$ with $L_{\mathbf{Q}}$, and hence we can view $\pi$ as a homomorphism into $L_{\mathbf{Q}}$. Since $\Gamma$ is finitely generated, there is a finite set of rational primes such that all denominators of all $\pi(\Gamma)$ have all prime factors in $S$. By diagonally embedding $L_{\mathbf{Q}}$, we obtain a representation $\sigma: \Gamma \rightarrow \widehat{L}=L_{\mathbf{R}} \times \prod_{p \in S} L_{\mathbf{Q}_{p}}$. By construction, $\operatorname{ker}(\sigma)=\operatorname{ker}(\pi)$, and it is standard that $\sigma(\Gamma)$ is discrete. Let $\alpha: M \times \widetilde{G} \rightarrow \Gamma$ be the cocycle given by the action on the universal cover. By Theorem 2.2, $\sigma \circ \alpha$ is equivalent to a cocycle taking all values in a compact subgroup $K \subset \widehat{L}$, and this implies by Lemma 2.3 that the Mackey range $X$ of $\alpha$ admits a measurable $\Gamma$-map $\theta: X \rightarrow \widehat{L} / K$. Since $\sigma(\Gamma)$ is discrete and $K$ is compact, the action of $\Gamma$ on $\hat{L} / K$ is tame. Since the action of $\Gamma$ on $X$ is ergodic, it follows that the image of $\theta$ lies in a single $\Gamma$-orbit; i.e., we can view $\theta$ as a measurable $\Gamma$-map $X \rightarrow \sigma(\Gamma) / F$ where $F$ is the intersection of $\sigma(\Gamma)$ with some conjugate of $K$. In particular, $F$ is finite. Letting $\Lambda=\sigma^{-1}(F)$ and $\Lambda_{0}=\operatorname{ker}(\sigma)$, we observe that if $\pi(\Gamma)$ is not finite, then $\Gamma / \Lambda_{0}$ is infinite, linear (and therefore residually finite [8]), and $\Lambda / \Lambda_{0}$ is finite. Since the action is engaging, this contradicts Proposition 3.5.

Theorem 5.2. Suppose $G$ is a connected semisimple Lie group each of whose simple factors has real rank at least 2 . Let $H$ be an algebraic $\bar{Q}$-group such that every homomorphism of Lie algebras $\mathfrak{g}_{\mathbf{C}} \rightarrow \mathfrak{h}$ is trivial. Assume there is an action of $G$ on $M$ that preserves a finite measure and is topologically engaging. If $\pi_{1}(M)$ is infinite, then there is no injective homomorphism $\pi_{1}(M) \rightarrow H_{\overline{\mathbf{Q}}}$.

Proof. The proof of Theorem 5.1 applies, and we will now have in addition that $\Lambda_{0}$ is trivial since we have an injective homomorphism. This contradicts the assumption of topological engaging by Proposition 3.6.

We now consider the situation in which $H$ is an algebraic group defined over $\mathbf{R}$, and we only assume there is nontrivial homomorphism $\mathfrak{g} \rightarrow \mathfrak{h}_{\mathbf{R}}$. To 
see the meaning of the distinction of this hypothesis from that of nonexistence of homomorphisms $\mathfrak{g} \rightarrow \mathfrak{h}$, let $H_{\mathbf{R}}$ be the Lorentz group $O(1, n)$. Then for any simple Lie group $G$ with $\mathbf{R}-\operatorname{rank}(G) \geq 2$, there is no nontrivial homomorphism $\mathfrak{g} \rightarrow \mathfrak{h}_{\mathbf{R}}$, but, of course, there may well be a nontrivial homomorphism $\mathfrak{g} \rightarrow \mathfrak{h}=$ $o(n+1, \mathbf{C})$. Thus, for example, the following theorems will give us much more information on actions on spaces with a fundamental group that embeds in a Lorentz group.

The following three theorems can be proven by a routine modification of the argument given in the proofs of Theorems 5.1 and 5.2.

Theorem 5.3. Suppose $G$ is a connected semisimple Lie group each of whose simple factors has real rank at least 2 . Let $H$ be an algebraic R-group such that every homomorphism of Lie algebras $\mathfrak{g} \rightarrow \mathfrak{h}_{\mathbf{R}}$ is trivial. Assume there is an action of $G$ on $M$ that preserves a finite measure.

(a) Suppose the action is engaging. Then every homomorphism $\pi_{1}(M) \rightarrow$ $H_{\mathbf{R}}$ with discrete image is finite.

(b) Suppose the action is topologically engaging and $\pi_{1}(M)$ is infinite. Then there is no injective homomorphism $\pi_{1}(M) \rightarrow H_{\mathbf{R}}$ with discrete image.

Theorem 5.4. Suppose $G$ is a connected semisimple Lie group each of whose simple factors has real rank at least 2 . Let $H$ be an algebraic Q-group such that every homomorphism of Lie algebras $\mathfrak{g} \rightarrow \mathfrak{h}_{\mathbf{R}}$ is trivial. Assume there is an action of $G$ on $M$ that preserves a finite measure.

(a) Suppose the action is engaging. Then every homomorphism $\pi_{1}(M) \rightarrow$ $H_{\mathbf{Q}}$ is finite.

(b) Suppose the action is topologically engaging and $\pi_{1}(M)$ is infinite. Then there is no injective homomorphism $\pi_{1}(M) \rightarrow H_{\mathbf{Q}}$.

Theorem 5.5. Suppose $G$ is a connected semisimple Lie group each of whose simple factors has real rank at least 2. Assume there is an action of $G$ on $M$ that preserves a finite measure.

(a) Suppose the action is engaging. Then every homomorphism of $\pi_{1}(M)$ into a product of finitely many p-adic groups with discrete image has finite image.

(b) Suppose the action is topologically engaging and $\pi_{1}(M)$ is infinite. Then $\pi_{1}(M)$ is not isomorphic to a discrete subgroup of a product of finitely many p-adic groups (where $p$ may vary).

For future reference, we also state a purely ergodic theoretic result, which follows by the same argument.

Theorem 5.6. Let $G$ be as in Theorem 5.1, and suppose that $S$ is an ergodic $G$-space with a finite invariant measure. Let $d(G)$ be the minimal dimension of a nontrivial (complex) representation of $g$. Suppose $\Lambda \subset \mathrm{GL}(n, \overline{\mathbf{Q}})$ is a finitely 
generated subgroup, where $n<d(G)$. If $\alpha: S \times G \rightarrow \Lambda$ is a cocycle, then $\alpha$ is equivalent to a cocycle into a finite subgroup of $\Lambda$.

\section{ON THE UNITARY DUAL OF $\pi_{1}(M)$}

It is not necessarily true that if there is an engaging or topologically engaging action of $G$ (which we assume to be as above, and, in particular, to satisfy Kazhdan's property (T)) on $M$ then $\pi_{1}(M)$ is also Kazhdan. Namely, suppose $\Gamma \subset G \times H$ is an irreducible lattice where $G$ is Kazhdan but $H$ is not. Such examples are easily constructed in $\mathrm{SO}(p, q) \times \mathrm{SO}(1, p+q-1)$ for instance. Then the action of $G$ on $M=(G \times H) / \Gamma$ is both engaging and topologically engaging, but as $\Gamma$ has a dense image homomorphism into a non-Kazhdan group, $\Gamma$ is not Kazhdan. On the other hand, the following remark shows that the identity is isolated in the space of finite unitary representations, i.e., those that factor through a finite quotient of $\Gamma$. These and related properties of a group have been discussed in a number of places, and we refer to [7] as an example and indication of other references.

Proposition 6.1. Suppose $G$ is a connected semisimple Kazhdan Lie group and that there is an engaging finite measure preserving action of $G$ on $M$. Then the identity representation of $\pi_{1}(M)$ is isolated in the space of finite representations of $\pi_{1}(M)$.

Proof. If not, we can choose finite quotients $p_{n}: \Gamma \rightarrow F_{n}$ and nontrivial irreducible representations $\left(\pi_{n}, V_{n}\right)$ of $F_{n}$ such that $\pi_{n} \circ p_{n} \rightarrow I$. Let $\alpha: M \times \widetilde{G} \rightarrow$ $\pi_{1}(M)$ be the cocycle defined by the action on the universal cover. We can suppose the measure on $M$ is ergodic. Form the unitary representation $\sigma_{n}$ of $\widetilde{G}$ on $L^{2}\left(M ; V_{n}\right)$ by $\left(\sigma_{n}(g) f\right)(m)=\pi_{n}\left(p_{n}(\alpha(m, g))\right) f(m g)$. Then $\left\{\sigma_{n}\right\}$ is readily seen to weakly contain $I$ (cf. [12, proof of 9.1.1]), and since $\widetilde{G}$ is Kazhdan, there is some $n$ for which $\sigma_{n}$ has a nonzero invariant vector. Since $\left(\pi_{n}, V_{n}\right)$ is contained in the regular representation of $F_{n}$, it is easy to see that this implies there is a nonconstant $\widetilde{G}$-invariant function in $L^{2}\left(M \times F_{n}\right)$, where the action of $\widetilde{G}$ on $M \times F_{n}$ is defined by the cocycle $\alpha$. This means the action on the finite cover of $M$ corresponding to $F_{n}$ is not ergodic, so that the action is not engaging.

\section{Amalgamated PRODUCTS}

Using a result of Adams and Spatzier [1], we can obtain further restrictions on fundamental groups of $G$-spaces. Namely, suppose $G$ is a Kazhdan group acting ergodically with a finite invariant measure on $S$. Let $H=H_{1}{ }^{*}{ }_{K} H_{2}$ be an amalgamated product of discrete groups. Then one of the main results of [1] asserts that any cocycle $\alpha: S \times G \rightarrow H$ is equivalent to a cocycle into $H_{1}$ 
or $\mathrm{H}_{2}$. We therefore can deduce

Theorem 7.1. Suppose $G$ is as in Theorem 5.1. Suppose, for $i=1,2$, that $\Gamma_{i}$ is a discrete group that is either amenable or isomorphic to an infinite finitely generated subgroup of $\mathrm{GL}(n, \overline{\mathbf{Q}})$, where $n<d(G)$ (and the latter is as in Theorem 5.6). Then there is no engaging or topologically engaging action of $G$ on $M$ with a finite invariant measure for any $M$ with $\pi_{1}(M)=\Gamma_{1}{ }_{K} \Gamma_{2}$, where $K$ is some common subgroup.

We leave the other variants of this theorem to the reader. (Cf. [8] for some useful relevant information on amalgamated products.)

\section{ACTIONS OF DISCRETE GROUPS}

There are a number of problems that arise when one tries to extend some of the above results to the case in which we assume only that there is an action of a lattice subgroup of $G$ rather than an action of $G$ itself. The first problem is considered in the following.

Definition 8.1. Suppose $\Gamma$ is a discrete group acting on $M$. We call the action admissible if there is a finite index subgroup $\Lambda \subset \Gamma$ so that the action of $\Lambda$ lifts to an action on $\widetilde{M}$, commuting with the action of $\pi_{1}(M)$.

Example 8.2. (a) The action of $\operatorname{SL}(n, Z)$ on $T^{n}$ by group automorphisms is not admissible, even though the action lifts.

(b) If the $\Gamma$ action extends to an action of a connected simply connected group, the action is admissible.

We shall also need some information on $H^{2}(\Gamma, \mathbf{R})$. Let us say that $\Gamma$ satisfies cohomology condition $(\mathrm{C})$ if $H^{2}(\Lambda, \mathbf{R})=0$ for every finite index subgroup $\Lambda \subset$ $\Gamma$. If the symmetric space associated to the semisimple group $G$ is Hermitian, then this will not be true, but in most other situations this will be the case. For example, it will be true for any cocompact lattice in $\operatorname{SL}(n, \mathbf{R})$ if $n>4$, and there are strong positive results for noncocompact lattices as well. In particular, this is true for $\operatorname{SL}(n, Z)$ for $n \geq 6$. See Borel's papers [2, 3], and Borel and Wallach [4] for an extensive discussion.

Proposition 8.3. Suppose $G$ is as in Theorem 5.1 and $\Gamma \subset G$ is a lattice satisfying condition (C). Let $d$ be the smallest dimension of a nontrivial representation of the Lie algebra of $G$. If $\Gamma$ acts on $M$ and $\pi_{1}(M)=Z^{n}$ for $n<d$, then the $\Gamma$ action is admissible.

Proof. Letting $D$ be the group of homeomorphisms of $\widetilde{M}$ obtained by taking all possible lifts of all elements of $\Gamma$ acting on $\widetilde{M}$, we obtain an extension $0 \rightarrow$ $\mathbf{Z}^{n} \rightarrow D \rightarrow \Gamma \rightarrow 0$. In particular, we have a homomorphism $\Gamma \rightarrow \operatorname{Aut}\left(\mathbf{Z}^{n}\right)=$ $\operatorname{SL}(n, \mathbf{Z})$. By Margulis superrigidity [12], since $n<d$ this homomorphism is trivial on a subgroup $\Lambda \subset \Gamma$ of finite index, and hence $0 \rightarrow Z^{n} \rightarrow D^{\prime} \rightarrow$ $\Lambda \rightarrow 0$ is a central extension, where $D^{\prime}$ is the inverse image of $\Lambda$. This is defined by an element of $H^{2}\left(\Lambda, \mathbf{Z}^{n}\right)$. Since $H^{2}(\Lambda, \mathbf{R})=0$, we have a surjection 
$H^{1}\left(\Lambda, T^{n}\right) \rightarrow H^{2}\left(\Lambda, \mathbf{Z}^{n}\right)$. Since $\Lambda$ is Kazhdan, every element of $H^{1}\left(\Lambda, T^{n}\right)$ is trivial on a subgroup of finite index, and hence this central extension is trivial on a subgroup of finite index.

We remark that we actually need assume only that $\pi_{1}(M)$ is finitely generated and abelian for this proof to work.

Definition 8.4. (a) Suppose the action of $\Gamma$ on $M$ is admissible. The action is called engaging if there is a lift of a finite index subgroup $\Lambda$ to $\widetilde{M}$ (commuting with the fundamental group) so that on every finite cover $M^{\prime} \rightarrow M$, all $\Lambda$ invariant measurable functions are lifted from $M$.

(b) Call the action on $M$ topologically engaging if there is some $\lambda \in \Lambda$ such that $\lambda$ acts tamely on $\widetilde{M}$ and has almost all ergodic components on $M$ properly ergodic. (That is, the ergodic components are not orbits. This will be the case, for example, if the $\Gamma$ action on $M$ is mixing; i.e., the matrix coefficients of the unitary representation on $L^{2}(M)$ vanish at $\infty$.)

Theorem 8.5. Let $G, \Gamma$, and $d$ be as in Proposition 8.3. Then there is no engaging or topologically engaging finite measure preserving action of $\Gamma$ on $M$ if $\pi_{1}(M)$ is an infinitely generated abelian group of rank less than $d$.

Proof. Every cocycle $\alpha: M \times \Gamma \rightarrow \pi_{1}(M)$ is equivalent to one into a finite subgroup, using Kazhdan's property [12,9.1.1]. This is incompatible with either engagement assumption.

In particular, Theorem 8.5 applies to actions of lattices in $\operatorname{SL}(n, \mathbf{Z})$ on tori of smaller rank, at least for $n$ sufficiently large. In this context this result has been independently established by $\mathrm{D}$. Witte (private communication).

\section{REFERENCES}

1. S. Adams and R. Spatzier, Kazhdan groups, cocycles, and trees (to appear).

2. A. Borel, Stable real cohomology of arithmetic groups, Ann. Sci. École Norm Sup. (4) 7 (1974), 235-272.

3. __ Stable real cohomology of arithmetic groups, II, Progr. Math., Vol. 15, Birkhauser, Boston, 1981, pp. 21-55.

4. A. Borel and N. Wallach, Continuous cohomology, discrete subgroups, and representations of reductive groups, Ann. of Math. Stud., Vol. 94, Princeton Univ. Press, Princeton, NJ, 1980.

5. M. Gromov, Rigid transformation groups (to appear).

6. F. E. A. Johnson, On the existence of irreducible discrete subgroups in isotypic Lie groups of classical type, Proc. London Math. Soc. 56 (1988), 51-77.

7. A. Lubotzky and R. J. Zimmer, Variants of Kazhdan's property for subgroups of semisimple groups, Israel J. Math. (to appear).

8. W. Magnus, Residually finite groups, Bull. Amer. Math. Soc. 75 (1969), 306-316.

9. R. J. Zimmer, Induced and amenable actions of Lie groups, Ann. Sci. École Norm. Sup. (4) 11 (1978), 407-428.

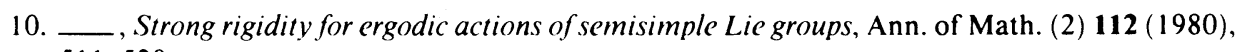
511-529. 
11. __, Orbit equivalence and rigidity of ergodic actions of Lie groups, Ergodic Theory Dynamical Systems 1 (1981), 237-253.

12. __ Ergodic theory and semisimple groups, Birkhauser, Boston, 1984.

13. __ Ergodic theory and the automorphism group of a G-structure, Group representations, ergodic theory, operator algebras, and mathematical physics (C. C. Moore, ed.), Springer, New York, 1987, pp. 247-278.

14. __ Arithmeticity of holonomy groups of Lie foliations, J. Amer. Math. Soc. 1 (1988), 35-58.

Department of Mathematics, University of Chicago, Chicago, Illinois 60637 